\title{
Population Pharmacokinetics of the Interleukin-23 Inhibitor Risankizumab in Subjects with Psoriasis and Crohn's Disease: Analyses of Phase I and II Trials
}

\author{
Ahmed A. Suleiman ${ }^{1} \cdot$ Amit Khatri $^{2} \cdot$ Mukul Minocha $^{2} \cdot$ Ahmed A. Othman ${ }^{2}$
}

Published online: 20 August 2018

(c) The Author(s) 2018

\begin{abstract}
Background and Objectives Risankizumab is a humanized anti-interleukin-23 monoclonal antibody in development for the treatment of several inflammatory diseases. This work characterized the pharmacokinetics of risankizumab and evaluated covariates that may affect its exposures using phase I and II trial data in subjects with psoriasis and Crohn's disease.

Methods Plasma concentration measurements from a phase I study and a phase II study in subjects with psoriasis $(n=157$; single doses of $0.01-5 \mathrm{mg} / \mathrm{kg}$ intravenously, $0.25-1 \mathrm{mg} / \mathrm{kg}$ subcutaneously, and $18 \mathrm{mg}$ subcutaneously, and multiple doses of 90 and $180 \mathrm{mg}$ subcutaneously), and a phase II study in subjects with Crohn's disease ( $n=115$; doses of $200 \mathrm{or} 600 \mathrm{mg}$ intravenously every 4 weeks followed by $180 \mathrm{mg}$ subcutaneously every 8 weeks) were analyzed using non-linear mixedeffects modeling. The model was qualified using bootstrap and simulation-based diagnostics.

Results A two-compartment model with first-order absorption and elimination described the pharmacokinetics of risankizumab. Considering the body weight and baseline albumin central tendency differences between disease populations, risankizumab clearance, steady-state volume of distribution, and terminal-phase elimination half-life were estimated to be approximately $0.35 \mathrm{~L} /$ day, $11.7 \mathrm{~L}$, and 27 days, respectively, for a typical 90-kg subject with psoriasis with an albumin level of $42 \mathrm{~g} / \mathrm{L}$, and $0.31 \mathrm{~L} / \mathrm{day}, 8.45 \mathrm{~L}$, and 22 days, respectively, for a typical $65-\mathrm{kg}$ subject with Crohn's disease with an albumin level of $37 \mathrm{~g} / \mathrm{L}$. Risankizumab absolute subcutaneous bioavailability and absorption rate constant were $72 \%$ and 0.18 day $^{-1}$, respectively. Inter-individual variability for clearance was $37 \%$.

Conclusions Risankizumab displayed pharmacokinetic characteristics typical for an IgG1 monoclonal antibody with no apparent target-mediated disposition. Accounting for the effects of body weight and baseline albumin explained the small differences in the pharmacokinetics of risankizumab between psoriasis and Crohn's disease, with no further differences between the patient populations.
\end{abstract}

Electronic supplementary material The online version of this article (https://doi.org/10.1007/s40262-018-0704-z) contains supplementary material, which is available to authorized users.

Ahmed A. Othman

ahmed.othman@abbvie.com

1 Clinical Pharmacology and Pharmacometrics, AbbVie Deutschland GmbH \& Co. KG, Ludwigshafen am Rhein, Germany

2 Clinical Pharmacology and Pharmacometrics, AbbVie Inc., 1 North Waukegan Road, North Chicago, IL 60064, USA 


\section{Key Points}

A robust two-compartment model with dose- and timeindependent pharmacokinetic parameters was developed to characterize the disposition of risankizumab, an antiinterleukin-23 monoclonal antibody, in subjects with plaque psoriasis and Crohn's disease.

Of the factors evaluated (demographics, disease population, laboratory values, and anti-drug antibody development), only body weight and baseline albumin level were statistically correlated with risankizumab clearance. Body weight had a modest effect on risankizumab exposure while albumin had no meaningful impact.

After accounting for differences in body weight and baseline albumin levels between psoriasis and Crohn's disease patient populations, no significant differences in the pharmacokinetics of risankizumab were found between the populations.

\section{Introduction}

Interleukin (IL)-23 is a component of a complex cytokine network regulating the function of $\mathrm{T}$ helper 17 cells serving to protect against extracellular pathogens, but can also play a role in the pathogenesis of several autoimmune disorders [1-5]. Interleukin-23 and its downstream cytokine, IL-17, have been targeted using several therapeutic monoclonal antibodies (mAbs) for treating immune-mediated diseases, including psoriasis, psoriatic arthritis, and inflammatory bowel diseases, such as Crohn's disease and ulcerative colitis [6, 7]. Ustekinumab, an approved anti-IL-23/anti-IL-12 $\mathrm{mAb}$ that binds to the $\mathrm{p} 40$ subunit of IL-23 (shared subunit of IL-12 and IL-23), has been shown to be efficacious in treating psoriasis, psoriatic arthritis, and Crohn's disease [8]. Other IL-23 blockers include guselkumab, recently approved for treating psoriasis, [9] and tildrakizumab, [10] which is being developed for the same indication. Secukinumab and ixekizumab, both IL-17 inhibitors, were also efficacious against several autoimmune inflammatory disorders [11-14].

Risankizumab is a humanized IgG1 mAb with kappa light chains, targeting specifically the p19 subunit of IL-23 [15]. By blocking IL-23 from binding to its receptor, risankizumab inhibits IL-23-dependent cell signaling and the release of proinflammatory cytokines. In a phase I study in subjects with moderate-to-severe plaque psoriasis, a single dose of risankizumab resulted in substantial improvements in the Psoriasis Area and Severity Index (PASI) scores as early as the second week after dosing. In this study, $87 \%$ $(27 / 31)$ and $71 \%(22 / 31)$ of the subjects who received any single dose of risankizumab [intravenous (IV) $0.01-5 \mathrm{mg} / \mathrm{kg}$ or subcutaneous (SC) 0.25 or $1 \mathrm{mg} / \mathrm{kg}$ ] achieved a PASI 75 response at week 12 and 24, respectively, compared with $0 / 8(0 \%)$ and $1 / 8(13 \%)$ of the subjects who received placebo, respectively [16]. In a subsequent phase II study, 77\% (64/83) of the subjects in the pooled 90- and 180-mg SC (administered at weeks 0,4 , and 16) risankizumab group achieved a PASI 90 response at week 12, compared with $40 \%$ (16/40) of the subjects in the pooled 45- and 90-mg SC ustekinumab group [17]. In a phase II study in subjects with moderate-to-severe Crohn's disease, $30.5 \%$ (25/82) of the subjects who received IV induction doses of 200 or $600 \mathrm{mg}$ of risankizumab every 4 weeks achieved clinical remission at week 12 compared with $15.4 \%$ (6/39) who received placebo [18, 19]. These results, combined with the favorable safety profile of risankizumab supported further development of risankizumab in larger phase III trials.

Risankizumab has recently completed three phase III studies in subjects with psoriasis and had the primary timepoint readout from a fourth phase III trial [20, 21]. The recent phase III trials confirmed the efficacy of a risankizumab SC regimen of $150 \mathrm{mg}$ administered at weeks 0 and 4 and every 12 weeks thereafter in subjects with psoriasis and demonstrated the superior efficacy of risankizumab relative to both adalimumab and ustekinumab in the treatment of psoriasis. Risankizumab is also currently being evaluated in phase III clinical trials for the treatment of Crohn's disease, [22-24] phase IIb/III trials for the treatment of ulcerative colitis, [25, 26] and phase IIb trials for the treatment of psoriatic arthritis [27, 28].

In the present analyses, pharmacokinetic data of risankizumab from the subjects enrolled in the aforementioned phase I and II studies in psoriasis and Crohn's disease were pooled and analyzed using a population approach. The aims were to (1) characterize the pharmacokinetics of risankizumab, (2) determine the effect of clinically relevant patientspecific covariates on risankizumab exposures, and (3) evaluate the consistency of the pharmacokinetics of risankizumab between psoriasis and Crohn's disease populations.

\section{Methods}

\subsection{Clinical Studies}

Studies included in the analyses were conducted in accordance with Good Clinical Practice guidelines and the ethical principles that have their origin in the Declaration of Helsinki. The protocols and informed consent forms were approved by the institutional review boards or ethics committees and participants provided written informed consent before any study-related procedures were performed. 
Details of the study designs and eligibility criteria have been described elsewhere, [16-19] and a summary of the dosing regimens and pharmacokinetic sampling schemes is provided in Table 1. Briefly, Study 1 was a phase I, randomized, placebo-controlled, first-in-human proof-of-concept study of single ascending doses of risankizumab in subjects with moderate-to-severe plaque psoriasis [16], Study 2 was a phase II, randomized, active-controlled (against ustekinumab), dose-ranging study of multiple doses of risankizumab in subjects with moderate-to-severe plaque psoriasis, [17] and Study 3 was a phase II, randomized, placebo-controlled, three-period study of multiple doses of risankizumab in subjects with moderate-to-severe Crohn's disease [18, 19].

\subsection{Bioanalysis}

Plasma risankizumab concentrations were determined using a validated, enzyme-linked immunosorbent assay method performed at PPD Development LLC (Richmond, VA, USA). The assay detects free risankizumab using a polyclonal antirisankizumab antibody as a capture reagent and a biotinylated anti-risankizumab idiotype antibody as the detection reagent. The method is applicable for the quantitation of risankizumab within a nominal range of $5-100 \mathrm{ng} / \mathrm{mL}$, with a lower limit of quantification of $5 \mathrm{ng} / \mathrm{mL}$. Plasma samples above the upper limit of quantitation were diluted and re-assayed. Across studies, overall precision was high (\% coefficient of variation was $<16.9 \%$ ) and bias was low ( -7.10 to $0.0576 \%)$.

A titer-based, acid dissociation-bridging electrochemiluminescence immunoassay with a disease population-specific cut-off point was developed for the detection of anti-drug antibodies (ADAs) against risankizumab in human plasma, as previously described in detail [17]. Samples were first analyzed in a screening assay, and positive samples were analyzed in a confirmatory assay. The ADA assay had a sensitivity of $0.219 \mathrm{ng} / \mathrm{mL}$ and drug tolerance allowed detection of $0.7 \mathrm{ng} / \mathrm{mL}$ of a positive control in the presence of $5 \mu \mathrm{g} / \mathrm{mL}$ of risankizumab. The positive samples from the confirmatory assay underwent serial dilutions to determine the ADA titer. The samples from Studies 2 and 3 were also further characterized in a validated, cell-based neutralizing anti-drug antibody (NAb) assay (via assessment of IL-23-induced STAT3 phosphorylation), followed by a specificity assay (using a mAb against risankizumab as a positive control). The NAb assay was not conducted for Study 1.

\subsection{Population-Pharmacokinetic Analyses}

\subsubsection{Software, and Model Selection Criteria}

The pharmacokinetic model was developed using a nonlinear mixed-effects modeling approach with NONMEM software (version 7.4.1; ICON Development Solutions, Ellicott City, MD, USA) [29]. Model parameters were estimated using the first-order conditional estimation method with interaction between inter-individual variability (IIV) and residual variability (FOCE with $\eta-\varepsilon$ interaction). The objective function value (OFV), a goodness-of-fit statistic, was used to compare the fits of nested models, where the difference in the OFV $(\triangle \mathrm{OFV})$ for models being compared can serve as a likelihood ratio test approximately following a chisquared distribution. A parsimonious approach was used for model development, and the model with the least number of parameters that could adequately describe the data was selected. With the exception of the backward elimination process in the covariate search where $\alpha$ was set to $0.001, \alpha$ was set to 0.01 for all other steps. Perl Speaks NONMEM (version 4.6.0) [30] and R (version 3.4.0) [31] were used to assist with developing and evaluating the model.

\subsubsection{Model Development}

Based on visual examination of the data, a two-compartment model with a linear elimination process was selected as the starting model. The model (using the ADVAN4 subroutine in NONMEM) was parameterized in terms of clearance $(C L)$, volumes of central $\left(V_{\mathrm{c}}\right)$ and peripheral $\left(V_{\mathrm{p}}\right)$ compartments, inter-compartmental clearance $(Q)$, absolute bioavailability $(F)$, and a first-order absorption rate constant $\left(K_{\mathrm{a}}\right)$. One- and three-compartment models, and non-linear saturable elimination of risankizumab were also evaluated during model development.

Inter-individual variabilities on $C L, V_{\mathrm{c}}$, and $K_{\mathrm{a}}$ were assumed to follow log-normal distributions and exponential random-effects error models were used for their description (Eq. 1):

$\theta_{i, k}=\theta_{k} * e^{\eta_{i, k}}$

where $\theta_{i, k}$ is the estimate for the $k$ th parameter for the $i$ th subject, $\theta_{k}$ is the typical population estimate of the $k$ th parameter, and $\eta_{i, k}$ is the parameter for individual deviation from $\theta_{k}$, and $\eta_{i, k}$ was assumed to be normally distributed with a mean of 0 and a variance of $\omega_{k}^{2}: \eta \sim N\left(0, \omega_{k}^{2}\right)$.

Inter-individual variability was tested on all parameters, but only included in the model if its incorporation rendered a significant reduction in the OFV and the overall robustness of the model was not compromised. Additive, proportional, and combined error models (combined error model depicted in Eq. 2) were tested and compared for the best description of the residual unexplained variability.

$C_{i j}=\hat{C}_{i j} *\left(1+\varepsilon_{1, i j}\right)+\varepsilon_{2, i j}$

where $C_{i j}$ is the observed risankizumab plasma concentration of the $i$ th individual at time $j, \hat{C}_{i j}$ is the corresponding 


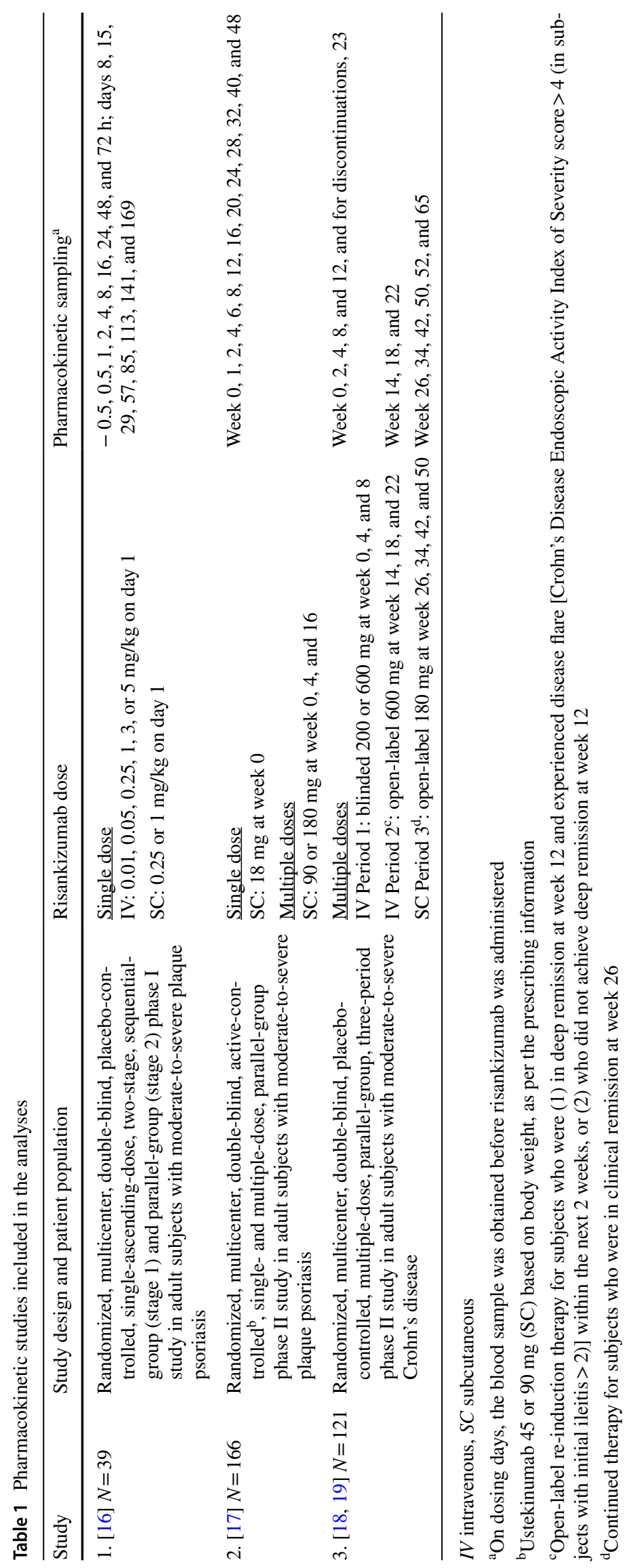


model-predicted risankizumab concentration, and $\varepsilon_{1, i j}$ and $\varepsilon_{2, i j}$ represent the proportional and additive residual random errors, respectively. These residual random errors were assumed to be independently normally distributed with a mean of 0 and a variance of $\sigma_{n}^{2}: \varepsilon \sim N\left(0, \sigma_{n}^{2}\right)$, where $n$ represents 1 (proportional) or 2 (additive) model structures in the combined error model.

Once the base model was developed, the well-established influence of body weight on the disposition of mAbs [32] was introduced into the model parameters $\left(C L, Q, V_{\mathrm{c}}\right.$, and $V_{\mathrm{p}}$ ) and evaluated for its significance. The parameterization for the effect on $C L$ as an example is depicted in Eq. (3):

$C L=C L_{70 \mathrm{~kg}} *(W T / 70)^{C L_{W T}}$

where $C L_{70 \mathrm{~kg}}$ is the typical population estimate of $C L$ for a reference $70-\mathrm{kg}$ individual, $W T$ is the body weight $(\mathrm{kg})$, and $C L_{\mathrm{WT}}$ is the exponent estimate for the power model characterizing the effect of body weight on $C L$. One exponent was estimated for both $C L$ and $Q$, and another for $V_{\mathrm{c}}$ and $V_{\mathrm{p}}$.

The effects of other covariates were investigated on the model parameters using the forward-inclusion $(\alpha=0.01)$ and backward-elimination $(\alpha=0.001)$ procedure as implemented using the automated stepwise covariates model building procedure in Pearl Speaks NONMEM. Relationships between key model parameters and continuous or categorical covariates were tested and included in the model if significant in a multiplicative manner as shown in Eq. (4):

$\theta_{i, k}=\theta_{k} *\left(\prod_{p=1}^{n_{p}}\left(\frac{\operatorname{cov}_{i, p}}{\operatorname{median}_{p}}\right)^{\theta_{k, p}} * \prod_{q=1}^{n_{q}}\left(1+\theta_{k, q, y}\right)\right) * e^{\left(\eta_{i, k}\right)}$

where $n_{p}$ is the number of continuous covariates, $\operatorname{cov}_{i, p}$ is the $p$ th continuous covariate value in the $i$ th subject, median $_{p}$ is the median value for the $p$ th covariate, and $\theta_{k, p}$ is the exponent estimate for the power model characterizing the effect of the $p$ th continuous covariate on the $k$ th parameter.

Similarly, $n_{q}$ is the number of categorical covariates and $\theta_{k, q, y}$ is the proportional difference estimate for the effect of the $y$ th category of the $q$ th categorical covariate on the $k$ th parameter, where $\theta_{k, q, y}$ takes a value of 0 for the most frequent category.

The impacts of ADAs and NAb on risankizumab $C L$ were evaluated as binary covariates (a flag for ADA or NAb-positive or -negative subjects, where subjects developing antibodies are flagged at all timepoints irrespective of the time of the antibody detection), as well as time-varying covariates (ADA titer values as a continuous variable or the time course of binary ADA or NAb positivity status with last observation carried forward imputation). Furthermore, patient population (i.e., psoriasis vs. Crohn's disease), age, sex, and laboratory values measured at baseline, including $\mathrm{C}$-reactive protein, serum albumin, alanine transaminase, aspartate transaminase, serum creatinine, as well as estimated creatinine clearance, were evaluated on risankizumab $C L$. Disease, age, and sex were tested on $V_{c}$, whereas the latter two covariates were tested on Ka. After covariate selection was finalized, covariance between $C L$ and $V_{\mathrm{c}}$ was evaluated using the OMEGA BLOCK statement in NONMEM.

\subsubsection{Model Evaluation}

Goodness-of-fit plots were used to evaluate the adequacy of the model in predicting observed data. Non-parametric bootstrap analysis was used to evaluate the model robustness, precision, and bias of parameter estimates. Using the original dataset, 1000 replicate datasets were generated by randomly sampling subjects with replacement. Model parameters were then estimated using the bootstrap datasets. The medians and the corresponding non-parametric 95\% confidence intervals ( 2.5 th-97.5th percentiles) were constructed for each parameter based on the successfully converging runs.

A visual predictive check was used to assess the predictive performance of the model. The final model parameters were used to simulate 1000 replicates of the observed data using NONMEM. The $95 \%$ confidence intervals of the median, 5th, and 95th percentiles of the simulated data representing the $90 \%$ prediction intervals were calculated and compared graphically with the observed data.

\subsubsection{Simulations}

The finalized pharmacokinetic model was used to simulate the risankizumab plasma concentration-time profiles for doses being evaluated for the respective indications: SC $150 \mathrm{mg}$ at weeks 0 and 4 , and every 12 weeks thereafter for psoriasis, [33-36] and an induction regimen of IV $600 \mathrm{mg}$ at weeks 0,4 , and 8 followed by a maintenance regimen of SC $180 \mathrm{mg}$, every 8 weeks starting week 12 for Crohn's disease. For each of the patient populations, pharmacokinetic profiles for 100,000 virtual subjects were simulated. Covariates for these subjects were sampled from distributions characterized by descriptive statistics of the respective observed covariates in each of the populations.

\section{Results}

\subsection{Data, Patient Demographics, and Baseline Characteristics}

Risankizumab plasma concentrations below the lower limit of quantification (169 out of 3000 samples; 5.63\%) were excluded from the analyses, leaving 2831 measurements 
from 272 individuals, of whom, 157 (57.7\%) were subjects with psoriasis who had a median baseline PASI score of 16.2 (10.5-61), and 115 (42.3\%) were subjects with Crohn's disease who had a median baseline Crohn's Disease Activity Index Score of 297 (109-518). A total of 152 (55.9\%) subjects were male and $120(44.1 \%)$ were female. Overall, subjects were predominantly white $(n=242 ; 89.0 \%)$, with a median (range) age of 43 years (19-74 years), and body weight of $79 \mathrm{~kg}(36-138 \mathrm{~kg})$. A summary of the demographics and baseline characteristics is provided in Table 2.

\subsection{Population-Pharmacokinetic Model}

The starting model was a two-compartment model with $F$ and $K_{\mathrm{a}}$ for SC administration and with IIV included on $C L$, $V_{\mathrm{c}}$, and $K_{\mathrm{a}}$. One- and three-compartment models and the addition of a non-linear elimination process were not found to be superior relative to a two-compartment model with only a linear elimination component.

Further inclusion of IIV on the peripheral compartment $V_{\mathrm{p}}$ rendered a reduction in the OFV by 178 points, while including IIV on $Q$ compromised the model robustness. Residual variability was best described by a proportional error model. Inclusion of body weight (normalized to $70 \mathrm{~kg}$ ) as a covariate on $C L$ and $V_{\mathrm{c}}$ led to a reduction in the OFV by
84 points, and a further reduction by 73 points was achieved by including body weight as a covariate on $Q$ and $V_{\mathrm{p}}$ (using the same exponents for $C L$ and $V_{\mathrm{c}}$, respectively).

In addition to body weight, only baseline serum albumin was identified as a significant covariate for risankizumab CL during the covariate forward-selection and backwardelimination search process $[\Delta \mathrm{OFV}=-56$; results of the univariate inclusion of the covariates are shown in the table in the Electronic Supplementary Material (ESM)]. Finally, introducing a correlation between $C L$ and $V_{\mathrm{c}}$ reduced the OFV by 135 points. Parameter estimates from the final population-pharmacokinetic model along with their associated relative standard errors are provided in Table 3.

Using a one-at-a-time elimination of covariate relationships from the final model, body weight and serum albumin appeared to explain 26 and $19 \%$, respectively, of the variance estimate for inter-subject variability in risankizumab $C L$. Additionally, body weight appeared to explain 65 and $24 \%$ of the variance estimate for inter-subject variability for risankizumab $V_{\mathrm{c}}$ and $V_{\mathrm{p}}$ respectively.

Based on the population-pharmacokinetic model and considering the body weight central tendency differences between disease populations, risankizumab $C L$, $V_{\mathrm{c}}, V_{\mathrm{p}}$, steady-state volume of distribution $\left(V_{\mathrm{c}}+V_{\mathrm{p}}\right)$, and terminal-phase elimination half-life were estimated to be

Table 2 Demographics and baseline characteristics of the subjects included in the risankizumab population-pharmacokinetic analysis

\begin{tabular}{|c|c|c|c|c|}
\hline Characteristic & Study $1(n=31)$ & Study $2(n=126)$ & Study $3(n=115)$ & Overall $(n=272)$ \\
\hline Population & Psoriasis & Psoriasis & Crohn's disease & \\
\hline \multicolumn{5}{|l|}{ Sex, $n(\%)$} \\
\hline Male & $25(80.7)$ & $82(65.1)$ & $45(39.1)$ & $152(55.9)$ \\
\hline Female & $6(19.3)$ & $44(34.9)$ & $70(60.9)$ & $120(44.1)$ \\
\hline \multicolumn{5}{|l|}{ Race, $n(\%)$} \\
\hline White & $28(90.3)$ & $117(92.9)$ & $97(84.3)$ & $242(89.0)$ \\
\hline Black & $2(6.5)$ & $4(3.1)$ & $1(0.9)$ & $7(2.6)$ \\
\hline Hispanic & $0(0.0)$ & $2(1.6)$ & $17(14.8)$ & $19(7.0)$ \\
\hline Asian & $1(3.2)$ & $1(0.8)$ & $0(0.0)$ & $2(0.7)$ \\
\hline American Indian/Alaska Native & $0(0.0)$ & $2(1.6)$ & $0(0.0)$ & $2(0.7)$ \\
\hline Age, year, median (range) & $45(24-61)$ & $47(20-72)$ & $35(19-74)$ & $42.5(19-74)$ \\
\hline Body weight, kg, median (range) & $89(47-121)$ & $89(49-138)$ & $66(36-126)$ & $79(36-138)$ \\
\hline \multicolumn{5}{|l|}{ Treatment-emergent ADAs, $n(\%)$} \\
\hline No & $28(90.3)$ & $108(85.7)$ & $106(92.2)$ & $242(89.0)$ \\
\hline Yes & $3(9.7)$ & $18(14.3)$ & $9(7.8)$ & $30(11.0)$ \\
\hline Albumin, $\mathrm{g} / \mathrm{L}$, median (range) & NA & $42(34-51)$ & $37(24-46)$ & $40(24-51)$ \\
\hline C-reactive protein, $\mathrm{mg} / \mathrm{L}$, median (range) & $3.3(0.3-34.4)$ & $2.6(0.2-30.0)$ & $10.0(0.2-146.0)$ & $4.3(0.2-146.0)$ \\
\hline Baseline PASI score, median (range) & $16.3(10.5-43.4)$ & $16.2(11.9-61)$ & NA & $16.2(10.5-61)^{\mathrm{a}}$ \\
\hline Baseline CDAI score, median (range) & NA & NA & $297(109-518)$ & $297(109-518)^{\mathrm{a}}$ \\
\hline
\end{tabular}

Subjects who did not receive at least a single risankizumab dose (treated with either placebo or ustekinumab) were not included in the analyses ADAs anti-drug antibodies, CDAI Crohn's Disease Activity Index, NA not applicable, PASI Psoriasis Area and Severity Index

${ }^{a}$ Disease severity scores (PASI and CDAI) have been summarized for the relevant populations only 
Table 3 Fixed- and random-effects parameter estimates for the risankizumab final population-pharmacokinetic model, and results of the bootstrap evaluation analysis

\begin{tabular}{lcc}
\hline Parameter & Estimate $(\% \mathrm{RSE})$ & Bootstrap median $^{\mathrm{a}}(95 \% \mathrm{CI})$ \\
\hline Clearance $(C L ; \mathrm{L} /$ day $)$ & $0.30(3)$ & $0.30(0.27-0.33)$ \\
Central volume of distribution $\left(V_{\mathrm{c}} ; \mathrm{L}\right)$ & $5.66(7)$ & $5.67(4.22-7.68)$ \\
Inter-compartmental $C L(Q ; \mathrm{L} / \mathrm{day})$ & $0.33(7)$ & $0.32(0.26-0.42)$ \\
Peripheral volume of distribution $\left(V_{\mathrm{p}} ; \mathrm{L}\right)$ & $3.43(5)$ & $3.40(2.73-3.95)$ \\
Absorption rate constant $\left(K_{\mathrm{a}} ;\right.$ day $\left.^{-1}\right)$ & $0.18(8)$ & $0.18(0.12-0.33)$ \\
Absolute $\mathrm{SC}$ bioavailability $(F)$ & $0.72(2)$ & $0.72(0.63-0.82)$ \\
Exponent for the effect of body weight on risankizumab $C L$ parameters $(C L$ and $Q)\left(C L_{\mathrm{WT}}\right)$ & $0.93(10)$ & $0.92(0.73-1.11)$ \\
Exponent for the effect of body weight on risankizumab volume of distribution parameters $\left(V_{\mathrm{c}}\right.$ & $0.99(11)$ & $0.97(0.78-1.18)$ \\
$\quad$ and $\left.V_{\mathrm{p}}\right)$ & $-1.54(8)$ & $-1.51(-1.84$ to -1.18$)$ \\
Exponent for the effect of serum albumin on risankizumab $C L$ & $37(4)$ & $37(32-42)$ \\
Inter-individual variability for $C L(\mathrm{IIV} C L ; \%)^{\mathrm{b}}$ & $55(6)$ & $55(42-66)$ \\
Inter-individual variability for volume of central compartment $\left(\mathrm{IIV} V_{\mathrm{c}} ; \%\right)^{\mathrm{b}}$ & $35(5)$ & $35(13-55)$ \\
Inter-individual variability for volume of peripheral compartment $\left(\mathrm{IIV} V_{\mathrm{p}} ; \%\right)^{\mathrm{b}}$ & $80(5)$ & $78(37-137)$ \\
Inter-individual variability for absorption rate constant $\left(\mathrm{IIV} K_{\mathrm{a}} ; \%\right)^{\mathrm{b}}$ & \\
\hline
\end{tabular}

$C I$ confidence interval, $R S E$ relative standard error, $S C$ subcutaneous

${ }^{a}$ Based on 989/1000 successful bootstrap runs

${ }^{\mathrm{b}} \mathrm{IIV}$ values are expressed in terms of coefficients of variation $(\% \mathrm{CV}) . \% \mathrm{CV}=\mathrm{SQRT}[\exp (\omega 2)-1] * 100$

approximately $0.35 \mathrm{~L} /$ day, $7.26,4.39,11.7 \mathrm{~L}$, and 27 days, respectively, for a typical $90-\mathrm{kg}$ subject with psoriasis with an albumin level of $42 \mathrm{~g} / \mathrm{L}$, and $0.31 \mathrm{~L} /$ day, 5.26, 3.19, $8.45 \mathrm{~L}$, and 22 days, respectively, for a typical $65-\mathrm{kg}$ subject with Crohn's disease with an albumin level of $37 \mathrm{~g} / \mathrm{L}$. The coefficient of variations for risankizumab $C L, V_{\mathrm{c}}, V_{\mathrm{p}}$, and $K_{\mathrm{a}}$ were $37,55,35$, and $80 \%$, respectively.

\subsection{Model Evaluation}

Figure 1 shows the goodness-of-fit-plots of the final model. The population predictions did not show a systematic bias across the evaluated concentration range and individual predictions adequately described the observed data (Fig. 1a, b and Fig. 1a, b of the ESM). Conditional weighted residuals were reasonably homogenously scattered around the null ordinates when plotted against the population predictions and time with no obvious trends in either plot (Fig. 1c, d).

Out of 1000 replicates in the bootstrap analysis, 989 runs converged successfully. Based on the successful runs, parameters were precisely estimated as indicated by the $95 \%$ confidence intervals, with negligible deviation from the original dataset estimates (Table 3). Visual predictive checks for the final model stratified by dose level and population (subjects with psoriasis or Crohn's disease) are presented in Figs. 2 and 3, respectively. These figures demonstrate the adequate predictive performance of the pharmacokinetic model.

\subsection{Simulations}

Figures 2 and 3 of the ESM illustrate the model-predicted concentrations and the inter-individual variability expected for the doses investigated in phase III trials in subjects with psoriasis (150 mg at weeks 0 and 4 and every 12 weeks thereafter) and the Crohn's disease efficacious regimen based on phase II (induction regimen of IV $600 \mathrm{mg}$ at weeks 0,4 , and 8 followed by a maintenance regimen of SC $180 \mathrm{mg}$ every 8 weeks starting at week 12). In subjects with psoriasis, the mean \pm standard deviation model-predicted risankizumab trough plasma concentrations were $1.72 \pm 1.11$ and $1.36 \pm 0.923 \mu \mathrm{g} / \mathrm{mL}$ at weeks 16 and 52 , respectively. Risankizumab area under the curve at steady state (weeks $40-52$ ) was predicted to be $344 \pm 151 \mu \mathrm{g} \cdot \mathrm{day} / \mathrm{mL}$.

Similarly, in subjects with Crohn's disease, the mean \pm standard deviation model-predicted risankizumab trough plasma concentrations were $33.9 \pm 18.1$ and $3.29 \pm 2.32 \mu \mathrm{g} / \mathrm{mL}$ at weeks 12 (induction phase) and 52 (maintenance phase), respectively. Risankizumab area under the curve at steady state (weeks 44-52) was predicted to be $433 \pm 206 \mu \mathrm{g} \cdot \mathrm{day} / \mathrm{mL}$.

Based on the simulations, and considering the identified statistically significant covariates (Fig. 4), body weight had a modest effect on risankizumab exposures for both populations, where relatively heavier subjects with body weights $>100 \mathrm{~kg}$ for psoriasis and $>80 \mathrm{~kg}$ for subjects with Crohn's disease were estimated to have 25 and $27 \%$ lower areas under the curve, respectively, compared with relatively lighter subjects. When comparing exposures of subjects with 

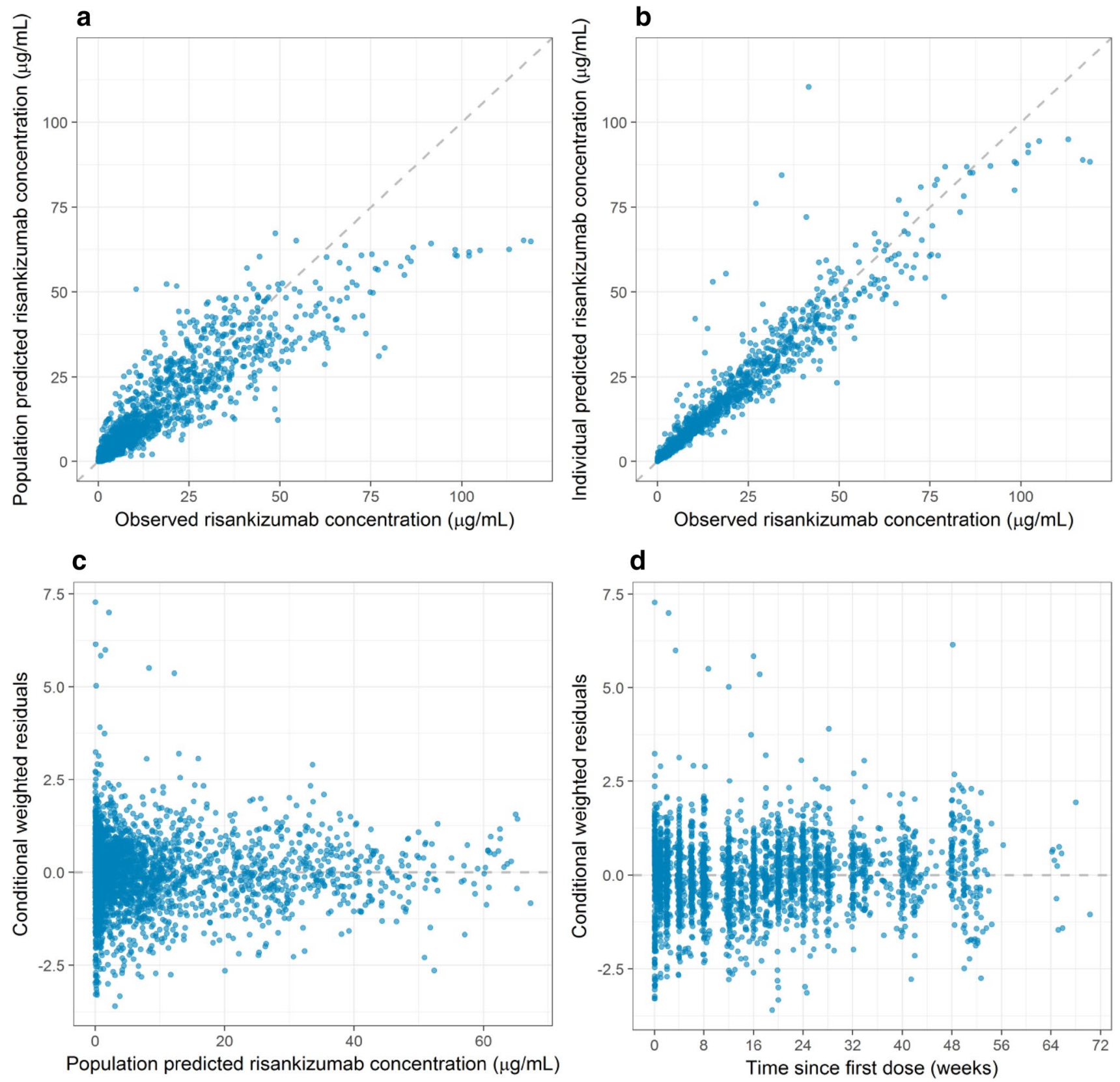

Fig. 1 Goodness-of-fit plots for the final population-pharmacokinetic model of risankizumab. a Population-predicted vs. observed risankizumab plasma concentrations; $\mathbf{b}$ individual-predicted vs. observed

risankizumab plasma concentrations; c conditional weighted residuals vs. population-predicted concentrations; and $\mathbf{d}$ conditional weighted residuals vs. time after first dose

albumin levels less than or higher than the 25 th and 75 th percentiles, respectively, of the observed albumin levels to exposures in subjects with albumin levels between these limits, albumin showed no meaningful impact. This was evident from the $95 \%$ confidence intervals largely overlapping with the default $0.8-1.25$ equivalence boundaries for both patient populations.

\section{Discussion}

Risankizumab, an anti-IL-23 $\mathrm{mAb}$, is currently being developed for several immune-mediated inflammatory diseases, such as psoriasis and Crohn's disease, where it has shown promising results. In four registration phase III studies in subjects with moderate-to-severe plaque psoriasis, risankizumab met all endpoints, almost half of the subjects 


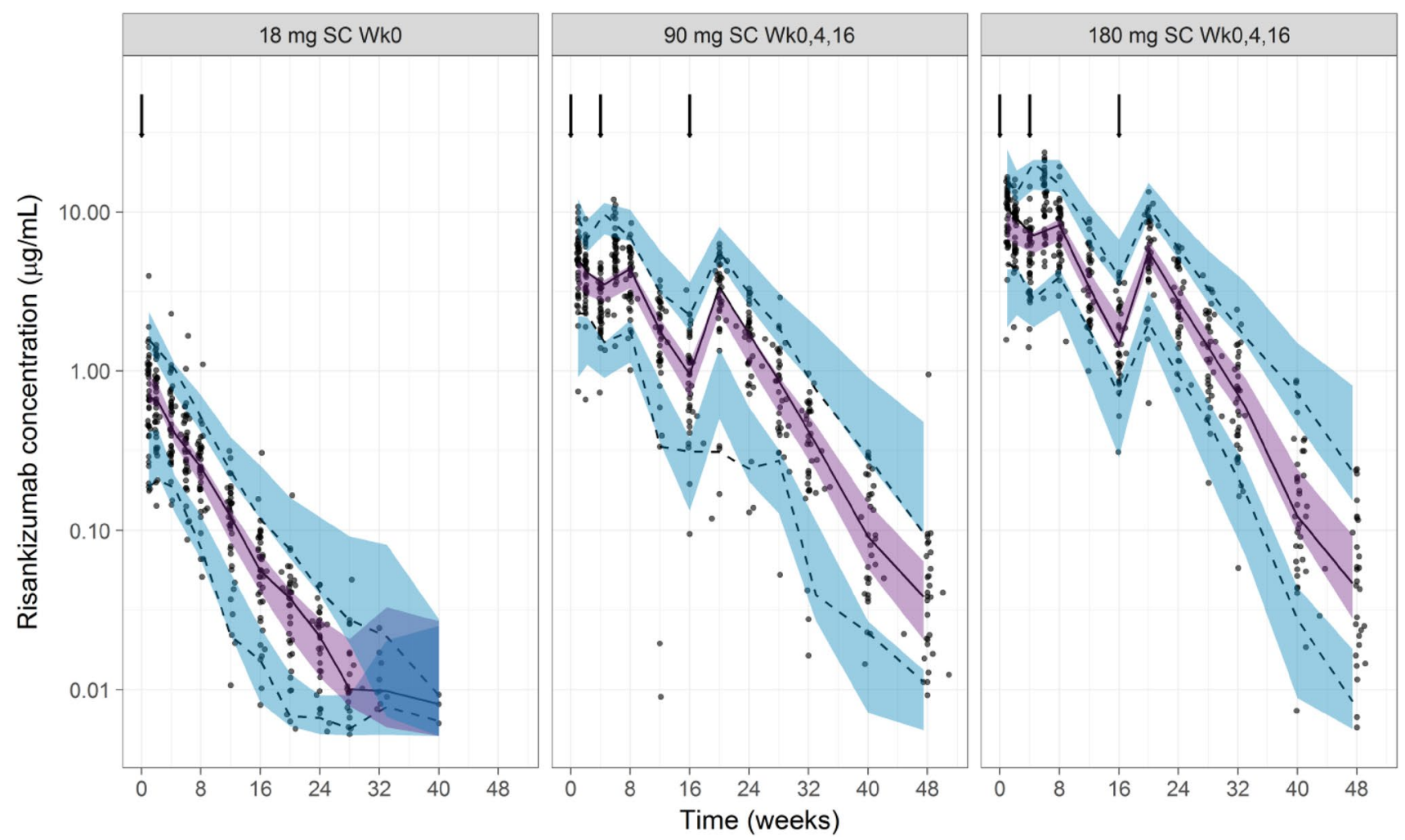

Fig. 2 Visual predictive check for the phase II psoriasis study using the final model. Solid lines represent the medians of the observed data; dashed lines represent the 5th and 95th percentiles of the observed data, together encompassing the $90 \%$ prediction interval;

achieved complete skin CL (PASI 100) and risankizumab demonstrated superior efficacy over adalimumab and ustekinumab [20, 21]. In a proof-of-concept study in subjects with Crohn's disease who mostly did not respond to prior tumor necrosis factor antagonist therapy, treatment with risankizumab resulted in significantly higher remission rates compared with placebo $[18,19]$. The current report summarizes the population pharmacokinetics of risankizumab in subjects with psoriasis and Crohn's disease using data from phase I and II clinical trials.

A two-compartment model with first-order absorption and elimination processes, with body weight and baseline albumin levels as the only identified statistically significant covariates, adequately characterized the pharmacokinetics of risankizumab as demonstrated by model diagnostics. Population- and individual-predicted risankizumab concentrations vs. the observed data suggested good model fits (Fig. 1a, b). Residual error model diagnostics showed no systematic trends and consistent distributions of the conditional weighted residuals vs. both risankizumab population predictions and time scales (Fig. 1c, d, respectively). Bootstrap analysis demonstrated the robustness of the model (Table 3). Furthermore, non-parametric 95\% confidence the shaded regions represent the $95 \%$ confidence intervals of the corresponding simulated percentiles (purple for the median and blue for the 5th and 95th percentiles); the arrows indicate the timings of the doses. $S C$ subcutaneous, $W k$ week

intervals showed high precision of the estimates, and medians were consistent with estimates based on the original dataset. Visual predictive checks demonstrated the predictive ability of the model to capture both the central tendency and the variability in the observed data across the evaluated risankizumab doses in subjects with psoriasis and Crohn's disease (Figs. 2 and 3, respectively).

Risankizumab displayed linear and dose- and time-independent pharmacokinetic characteristics across the dose range evaluated in the phase I and II studies included in these analyses. Based on body weight and albumin differences between psoriasis and Crohn's disease populations (subjects with psoriasis were relatively heavier with higher baseline albumin levels, Table 2), risankizumab CL, steadystate volume of distribution $\left(V_{\mathrm{c}}+V_{\mathrm{p}}\right)$, and terminal-phase elimination half-life were estimated to be approximately $0.35 \mathrm{~L} /$ day, $11.7 \mathrm{~L}$, and 27 days, respectively, for a typical 90-kg subject with psoriasis with an albumin level of $42 \mathrm{~g} / \mathrm{L}$, and $0.312 \mathrm{~L} /$ day, $8.45 \mathrm{~L}$, and 22 days, respectively, for a typical 65-kg subject with Crohn's disease with an albumin level of $37 \mathrm{~g} / \mathrm{L}$. These values are consistent with values typically reported for $\mathrm{IgG} \mathrm{mAbs}$ with no target-mediated disposition [32]. Furthermore, estimated elimination half-lives 

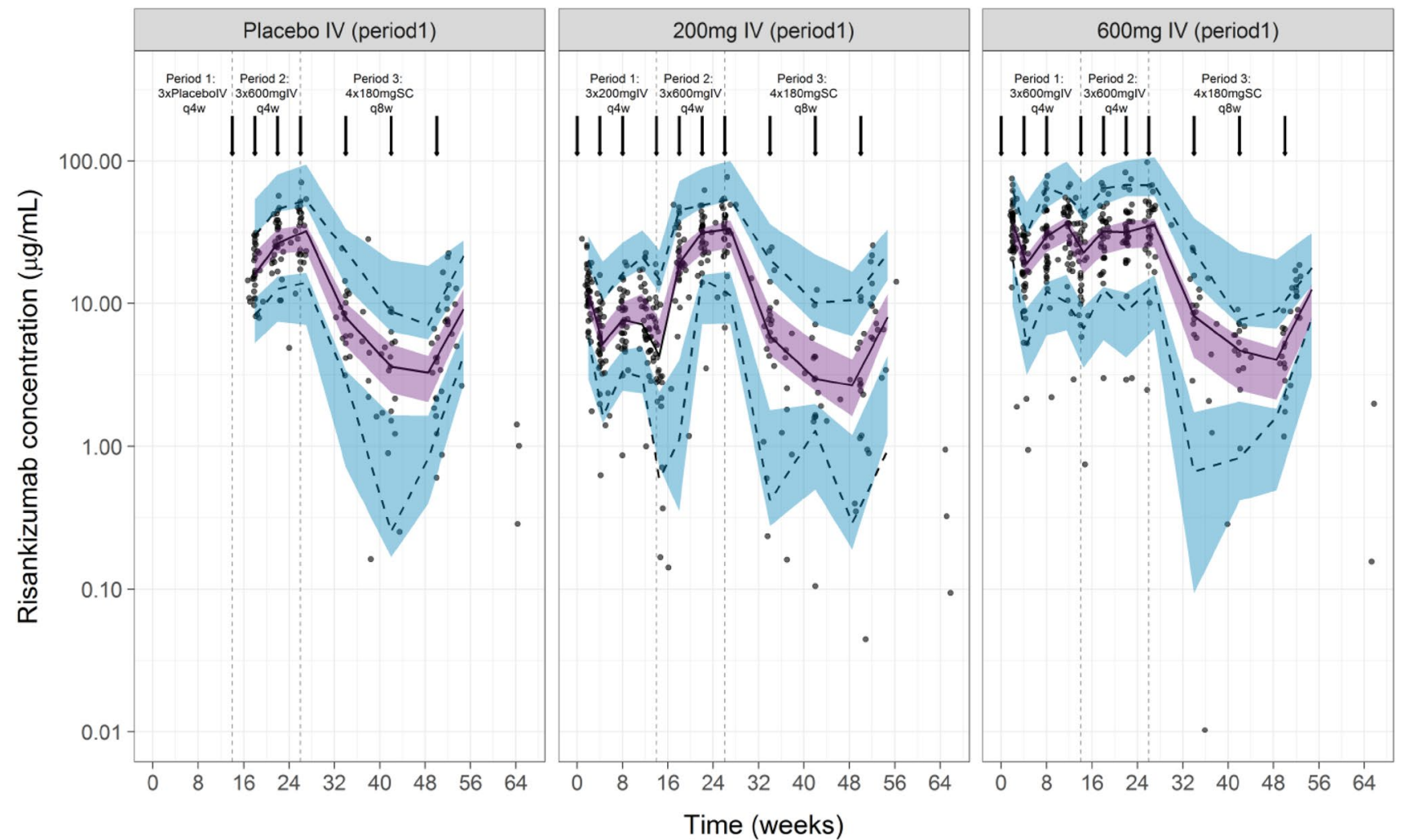

Fig. 3 Visual predictive check for the phase II Crohn's disease study using the final model. Solid lines represent the medians of the observed data; dashed lines represent the 5th and 95th percentiles of the observed data, together encompassing the $90 \%$ prediction interval; the shaded regions represent the $95 \%$ confidence intervals of the corresponding simulated percentiles (purple for the median and blue for the 5th and 95th percentiles); the arrows indicate the timings of the doses. $I V$ intravenous, $S C$ subcutaneous, $q 4 w$ every 4 weeks, $q 8 w$ every 8 weeks

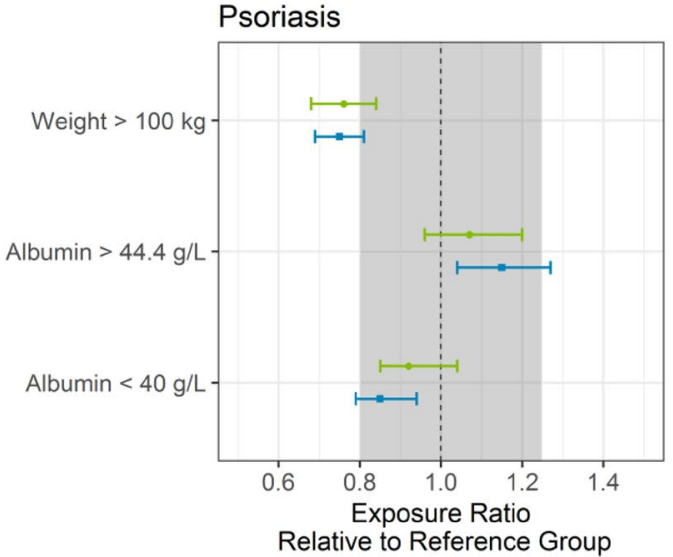

Fig. 4 Effect of statistically significant covariates on risankizumab simulated exposures in subjects with psoriasis (left) and Crohn's disease (right). Points represent medians and error bars represent $95 \%$ confidence intervals of the exposure ratios relative to the reference

were consistent with the elimination half-life estimates (20-28 days) based on non-compartmental analyses of the psoriasis phase I data [16]. Absolute SC bioavailability of

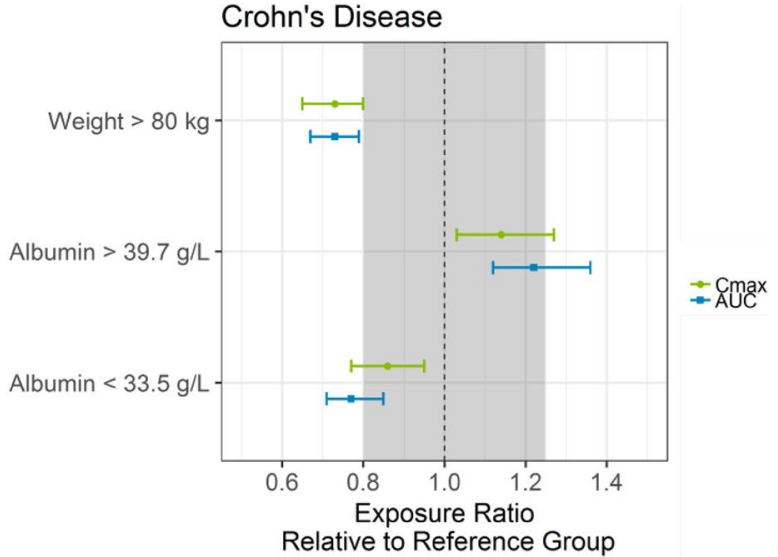

values across 200 simulation replicates, each with 500 virtual subjects. $A U C$ area under the curve, $C_{\max }$ maximum plasma concentration

risankizumab was estimated to be $72 \%$, and the first-order absorption rate constant following SC administration was 
estimated to be $0.18 \mathrm{day}^{-1}$, corresponding to an estimated mean absorption time of approximately 6 days.

A number of patient-specific covariates, including age, body weight, sex, various laboratory values (C-reactive protein, serum albumin, alanine transaminase, aspartate transaminase, serum creatinine), immunogenicity towards risankizumab, as well as disease populations (psoriasis vs. Crohn's disease) were evaluated for their effects on the disposition of risankizumab. As previously noted, only body weight and baseline albumin levels were correlated to the pharmacokinetic parameters of risankizumab, and explained the small differences between the patient populations. The exponents describing the effect of body weight on risankizumab CL and central volume of distribution in the model were estimated to be 0.93 and 0.99 , respectively. Similar relationships and exponents between body weight and pharmacokinetic parameters have also been reported for other therapeutic mAbs, such as ustekinumab, [37] daclizumab, [38] secukinumab, [39] and guselkumab [40]. This body weight effect translates into modest effects on risankizumab exposure as illustrated in Fig. 4. Risankizumab $C L$ was found to be inversely correlated with baseline serum albumin levels, albeit with no meaningful impact on the exposures in subjects with relatively low and high levels (Fig. 4). The correlation of albumin levels and $C L$ of $\mathrm{mAbs}$ is well documented [41, 42]. High albumin levels can be indicative of higher expression levels of the neonatal $\mathrm{Fc}$ receptors $(\mathrm{FcRn})$, which offer both albumin and immunoglobulins a protective mechanism against lysosomal catabolism by recycling them across the plasma membrane back to the circulatory system [32, 43]. Another potential reason for the inverse correlation could be loss of proteins in general (including albumin or therapeutic antibodies) as a result of leakage into the lumen of the large intestine through ulcerated intestinal mucosa in subjects with inflammatory bowel disease [44]. However, given that no further difference in the pharmacokinetic parameters of risankizumab was estimated between psoriasis and Crohn's disease patient populations across the evaluated studies when disease population was tested as a covariate, there is no evidence to suggest that intestinal leakage has a meaningful impact on risankizumab $C L$ in subjects with Crohn's disease.

The development of ADAs has been observed to affect the $C L$ of several mAbs [37, 45-47]. Across the three studies included in these analyses, $11 \%$ of the subjects developed ADAs following risankizumab administration. Based on the observed risankizumab plasma concentration data, no clear or consistent impact of ADA development on exposure to risankizumab was discernible in this relatively small dataset. This was further tested quantitatively by evaluating ADAs as a time-varying covariate for correlation with risankizumab $C L$ in the population-pharmacokinetic model, which in turn confirmed a lack of a statistically significant effect of ADAs on the disposition of risankizumab. Data from the larger ongoing phase III trials across indications will be more conclusive for the assessment of the potential impact of ADA or NAb development on risankizumab exposures. The unexplained IIV in $C L$ and volume of distribution parameters for risankizumab was comparable to that of other therapeutic mAbs (coefficients of variation estimates were 37 and 55\%, respectively).

The efficacy of risankizumab was evaluated following multiple-dose administration of SC $90 \mathrm{mg}$ and $180 \mathrm{mg}$ at weeks 0,4 , and 16 in the phase II study in subjects with psoriasis (Study 2). In this study, both doses demonstrated efficacy at week 12 , where $73 \%$ of the subjects in the $90-\mathrm{mg}$ dose group and $81 \%$ of the subjects in the $180-\mathrm{mg}$ dose group achieved PASI 90, respectively [17]. Exposure-response analyses of the data from this study suggested that a fixed-dose regimen irrespective of body weight for phase III evaluation would provide adequate exposures and comparable efficacy across the expected body weight range for this patient population (data on file, AbbVie). Phase III studies in subjects with moderate-to-severe psoriasis that evaluated SC risankizumab $150 \mathrm{mg}$ administered at weeks 0,4 , and every 12 weeks thereafter have recently concluded and reports of the phase III pharmacokinetic and exposure-response results in subjects with psoriasis are forthcoming. Figure 1 of the ESM shows the predicted risankizumab plasma concentrations following administration of the phase III regimen based on the phase I-II populationpharmacokinetic model described in this report.

Similarly, in the phase II study in subjects with Crohn's disease (Study 3), both risankizumab IV $200 \mathrm{mg}$ and risankizumab IV $600 \mathrm{mg}$ every 4 weeks over 12-24 weeks of induction therapy showed improvement in clinical remission at week 12 [10/41 (24\%) and 15/41 (37\%) of subjects, respectively] compared with placebo [6/39 (15\%)]. In this study, risankizumab showed a favorable safety profile; the most common adverse event was nausea and the most common serious adverse event was worsening of underlying Crohn's disease. No association was observed between risankizumab dose and the incidence of adverse events [18]. Detailed reports of the exposure-response analyses of the Crohn's disease phase IIb trials are planned.

This is the first report to present the population pharmacokinetics of risankizumab, currently in the late stage of development for several inflammatory diseases. This analysis provides quantitative assessments of the pharmacokinetic characteristics of risankizumab in subjects with psoriasis or Crohn's disease and characterizes the impact of patient characteristics on exposure to risankizumab. This analysis also presents the foundation for assessing the correlation between exposure to risankizumab and the efficacy of risankizumab in psoriasis and Crohn's disease. The findings based on this 
analysis warrant further confirmation using data from large, phase III clinical trials.

\section{Conclusions}

The pharmacokinetics of risankizumab were described using a two-compartment model with linear absorption, distribution, and elimination processes within the range of doses tested in subjects with psoriasis and Crohn's disease. Accounting for the effects of body weight and baseline albumin explained the small differences in the pharmacokinetics of risankizumab between psoriasis and Crohn's disease, with no further differences between the patient populations.

Acknowledgements The authors thank Allison Kitten, an employee of AbbVie, for her medical writing support.

\section{Compliance with Ethical Standards}

Funding This work was supported by Boehringer Ingelheim and AbbVie. Boehringer Ingelheim contributed to the study designs and data collection and AbbVie contributed to the analysis and interpretation of the data and the writing, review, and approval of the manuscript. Boehringer Ingelheim contributed to the approval of the manuscript.

Conflict of interest All authors are employees of AbbVie and may hold AbbVie stock or stock options.

Ethics Approval Studies included in the analyses were conducted in accordance with Good Clinical Practice guidelines and the ethical principles that have their origin in the Declaration of Helsinki. The protocols and informed consent forms were approved by the institutional review boards or ethics committees.

Consent to Participate Participants provided written informed consent before any study-related procedures were performed.

Open Access This article is distributed under the terms of the Creative Commons Attribution-NonCommercial 4.0 International License (http://creativecommons.org/licenses/by-nc/4.0/), which permits any noncommercial use, distribution, and reproduction in any medium, provided you give appropriate credit to the original author(s) and the source, provide a link to the Creative Commons license, and indicate if changes were made.

\section{References}

1. Patel DD, Kuchroo VK. Th17 cell pathway in human immunity: lessons from genetics and therapeutic interventions. Immunity. 2015;43(6):1040-51.

2. McGovern D, Powrie F. The IL23 axis plays a key role in the pathogenesis of IBD. Gut. 2007;56(10):1333-6.

3. Iwakura Y, Nakae S, Saijo S, Ishigame H. The roles of IL-17A in inflammatory immune responses and host defense against pathogens. Immunol Rev. 2008;226:57-79.
4. Bowes J, Barton A. The genetics of psoriatic arthritis: lessons from genome-wide association studies. Discov Med. 2010;10(52):177-83.

5. Smith RL, Warren RB, Eyre S, Ho P, Ke X, Young HS, et al. Polymorphisms in the IL-12beta and IL-23R genes are associated with psoriasis of early onset in a UK cohort. J Investig Dermatol. 2008;128(5):1325-7.

6. Fragoulis GE, Siebert S, McInnes IB. Therapeutic targeting of IL-17 and IL-23 cytokines in immune-mediated diseases. Annu Rev Med. 2016;67:337-53.

7. Neurath MF. Current and emerging therapeutic targets for IBD. Nat Rev Gastroenterol Hepatol. 2017;14(5):269-78.

8. Stelara (ustekinumab) [US prescribing information]. Horsham (PA): Janssen Biotech, Inc., 2016.

9. Tremfya (guselkumab) [US prescribing information]. Horsham (PA): Janssen Biotech, Inc., 2017.

10. Reich K, Papp KA, Blauvelt A, Tyring SK, Sinclair R, Thaci $\mathrm{D}$, et al. Tildrakizumab versus placebo or etanercept for chronic plaque psoriasis (reSURFACE 1 and reSURFACE 2): results from two randomised controlled, phase 3 trials. Lancet. 2017;390(10091):276-88.

11. Thaci D, Blauvelt A, Reich K, Tsai TF, Vanaclocha F, Kingo K, et al. Secukinumab is superior to ustekinumab in clearing skin of subjects with moderate to severe plaque psoriasis: CLEAR, a randomized controlled trial. J Am Acad Dermatol. 2015;73(3):400-9.

12. Gordon KB, Blauvelt A, Papp KA, Langley RG, Luger T, Ohtsuki M, et al. Phase 3 trials of Ixekizumab in moderate-to-severe plaque psoriasis. N Engl J Med. 2016;375(4):345-56.

13. Baeten D, Sieper J, Braun J, Baraliakos X, Dougados M, Emery $\mathrm{P}$, et al. Secukinumab, an interleukin-17A inhibitor, in ankylosing spondylitis. N Engl J Med. 2015;373(26):2534-48.

14. Mease PJ, McInnes IB, Kirkham B, Kavanaugh A, Rahman $P$, van der Heijde $D$, et al. Secukinumab inhibition of interleukin-17A in patients with psoriatic arthritis. N Engl J Med. 2015;373(14):1329-39.

15. Singh S, Kroe-Barrett RR, Canada KA, Zhu X, Sepulveda E, Wu $\mathrm{H}$, et al. Selective targeting of the IL23 pathway: generation and characterization of a novel high-affinity humanized anti-IL23A antibody. MAbs. 2015;7(4):778-91.

16. Krueger JG, Ferris LK, Menter A, Wagner F, White A, Visvanathan S, et al. Anti-IL-23A mAb BI 655066 for treatment of moderate-to-severe psoriasis: safety, efficacy, pharmacokinetics, and biomarker results of a single-rising-dose, randomized, double-blind, placebo-controlled trial. J Allergy Clin Immunol. 2015;136(1):116-24.

17. Papp KA, Blauvelt A, Bukhalo M, Gooderham M, Krueger JG, Lacour JP, et al. Risankizumab versus ustekinumab for moderateto-severe plaque psoriasis. N Engl J Med. 2017;376(16):1551-60.

18. Feagan BG, Sandborn WJ, D'Haens G, Panes J, Kaser A, Ferrante $\mathrm{M}$, et al. Induction therapy with the selective interleukin-23 inhibitor risankizumab in patients with moderate-to-severe Crohn's disease: a randomised, double-blind, placebo-controlled phase 2 study. Lancet. 2017;389(10080):1699-709.

19. Feagan BG, Panes J, Ferrante M, Kaser A, D’Haens GR, Sandborn WJ, et al. Risankizumab in patients with moderate to severe Crohn's disease: an open-label extension study. Lancet Gastroenterol Hepatol. 2018. https://doi.org/10.1016/S2468 -1253(18)30233-4.

20. Gordon KB, Strober B, Lebwohl M, Augustin M, Blauvelt A, Poulin Y, et al. Efficacy and Safety of risankizumab in moderateto-severe plaque psoriasis (UltIMMa-1 and UltIMMa-2): results from two double-blind, randomised, placebo-controlled and ustekinumab-controlled phase 3 trials. Lancet. 2018. https://doi. org/10.1016/S0140-6736(18)31713-6.

21. AbbVie. Press release: risankizumab meets all primary endpoints reporting positive results in fourth pivotal phase 3 psoriasis study. 
https://news.abbvie.com/news/risankizumab-meets-all-prima ry-endpoints-reporting-positive-results-in-fourth-pivotal-phase -3-psoriasis-study.htm. Accessed 9 Feb 2018.

22. AbbVie. A study to assess the efficacy and safety of risankizumab in subjects with moderately to severely active Crohn's disease who failed prior biologic treatment [ClinicalTrials.gov identifier: NCT03104413]. https://clinicaltrials.gov/ct2/show/NCT0310441 $3 \% 3$ Fterm $=$ NCT03104413\%26rank=1 Accessed 9 Feb 2018.

23. AbbVie. A study of the efficacy and safety of risankizumab in subjects with moderately to severely active Crohn's disease [ClinicalTrials.gov identifier: NCT03105128]. https://clinicaltrials.gov/ ct $2 /$ show $/$ NCT03105128\%3Fterm $=$ NCT03105128\%26rank $=1$. Accessed 9 Feb 2018.

24. AbbVie. A study of the efficacy and safety of risankizumab in subjects with Crohn's disease who responded to induction treatment in M16-006 or M15-991 [ClinicalTrials.gov identifier: NCT03105102]. https://clinicaltrials.gov/ct2/show/NCT0310510 $2 \% 3$ Fterm $=$ NCT03105102\%26rank=1 .Accessed 9 Feb 2018

25. AbbVie. A study to assess the efficacy and safety of risankizumab in subjects with ulcerative colitis who responded to induction treatment in M16-067 or M16-065 [ClinicalTrials.gov identifier: NCT03398135]. https://clinicaltrials.gov/ct2/show/NCT0339813 5\%3Fterm=NCT03398135\%26rank=1. Accessed 9 Feb 2018.

26. AbbVie. A study to evaluate the efficacy and safety of risankizumab in subjects with moderately to severely active ulcerative colitis who have failed prior biologic therapy [ClinicalTrials.gov identifier: NCT03398148]. https://clinicaltrials.gov/ct2/show/ NCT03398148\%3Fterm $=$ NCT03398148\%26rank $=1$. Accessed 9 Feb 2018.

27. AbbVie. BI 655066/ABBV-066/risankizumab compared to placebo in patients with active psoriatic arthritis [ClinicalTrials.gov identifier: NCT02719171]. https://clinicaltrials.gov/ct2/show/ NCT02719171\%3Fterm $=$ NCT02719171\%26rank=1. Accessed 9 Feb 2018.

28. AbbVie. A study to investigate safety with risankizumab in psoriatic arthritis subjects who have completed week 24 visit of Study 1311.5 [ClinicalTrials.gov identifier: NCT02986373]. https://clini caltrials.gov/ct2/show/NCT02986373\%3Fterm =NCT0298637 3\%26rank=1. Accessed 9 Feb 2018.

29. Beal S, Sheiner LB, Boeckmann A, Bauer RJ. NONMEM user's guides (1989-2017). Ellicott City (MD): Icon Development Solutions; 2017.

30. Lindbom L, Pihlgren P, Jonsson EN. PsN-Toolkit: a collection of computer intensive statistical methods for non-linear mixed effect modeling using NONMEM. Comput Methods Programs Biomed. 2005;79(3):241-57.

31. R Core Team. R: a language and environment for statistical computing. R Foundation for Statistical Computing, Vienna, Austria. 2017. https://www.R-project.org/. Accessed 25 Jul 2018.

32. Keizer RJ, Huitema AD, Schellens JH, Beijnen JH. Clinical pharmacokinetics of therapeutic monoclonal antibodies. Clin Pharmacokinet. 2010;49(8):493-507.

33. AbbVie. BI 655066 compared to placebo \& active comparator (ustekinumab) in patients with moderate to severe chronic plaque psoriasis [ClinicalTrials.gov identifier: NCT02684357]. https ://clinicaltrials.gov/ct2/show/NCT02684357\%3Fterm=NCT02 684357\%26rank=1. Accessed 9 Feb 2018.
34. AbbVie. BI 655066/ABBV-066 (risankizumab) compared to active comparator (adalimumab) in patients with moderate to severe chronic plaque psoriasis [ClinicalTrials.gov identifier: NCT02694523]. https://clinicaltrials.gov/ct2/show/NCT0269452 $3 \% 3$ Fterm $=$ NCT02694523\%26rank=1. Accessed $9 \mathrm{Feb} 2018$.

35. AbbVie. BI 655066 (risankizumab) compared to placebo and active comparator (ustekinumab) in patients with moderate to severe chronic plaque psoriasis [ClinicalTrials.gov identifier: NCT02684370]. https://clinicaltrials.gov/ct2/show/NCT0268437 0\%3Fterm $=$ NCT02684370\%26rank=1. Accessed 9 Feb 2018.

36. AbbVie. BI 655066/ABBV-066 (risankizumab) in moderate to severe plaque psoriasis with randomized withdrawal and re-treatment [ClinicalTrials.gov identifier: NCT02672852]. https://clini caltrials.gov/ct2/show/NCT02672852\%3Fterm $=$ NCT0267285 $2 \% 26$ rank=1. Accessed 9 Feb 2018.

37. Zhu Y, Hu C, Lu M, Liao S, Marini JC, Yohrling J, et al. Population pharmacokinetic modeling of ustekinumab, a human monoclonal antibody targeting IL-12/23p40, in patients with moderate to severe plaque psoriasis. J Clin Pharmacol. 2009;49(2):162-75.

38. Diao L, Hang Y, Othman AA, Nestorov I, Tran JQ. Population pharmacokinetics of daclizumab high-yield process in healthy volunteers and subjects with multiple sclerosis: analysis of phase I-III clinical trials. Clin Pharmacokinet. 2016;55(8):943-55.

39. Bruin G, Loesche C, Nyirady J, Sander O. Population pharmacokinetic modeling of secukinumab in patients with moderate to severe psoriasis. J Clin Pharmacol. 2017;57(7):876-85.

40. Yao Z, Hu C, Zhu Y, Xu Z, Randazzo B, Wasfi Y, et al. Population pharmacokinetic modeling of guselkumab, a human IgG1lambda monoclonal antibody targeting IL-23, in patients with moderate to severe plaque psoriasis. J Clin Pharmacol. 2018;58(5):613-27.

41. Rosario M, Dirks NL, Gastonguay MR, Fasanmade AA, Wyant T, Parikh A, et al. Population pharmacokinetics-pharmacodynamics of vedolizumab in patients with ulcerative colitis and Crohn's disease. Aliment Pharmacol Ther. 2015;42(2):188-202.

42. Fasanmade AA, Adedokun OJ, Olson A, Strauss R, Davis HM. Serum albumin concentration: a predictive factor of infliximab pharmacokinetics and clinical response in patients with ulcerative colitis. Int J Clin Pharmacol Ther. 2010;48(5):297-308.

43. Ryman JT, Meibohm B. Pharmacokinetics of monoclonal antibodies. CPT Pharmacometrics Syst Pharmacol. 2017;6(9):576-88.

44. Brandse JF, van den Brink GR, Wildenberg ME, van der Kleij $\mathrm{D}$, Rispens T, Jansen JM, et al. Loss of infliximab into feces is associated with lack of response to therapy in patients with severe ulcerative colitis. Gastroenterology. 2015;149(2):350-5.

45. Center for Drug Evaluation and Research. Clinical pharmacology and biopharmaceutics review for ixekizumab. Application number 125521Orig1s000. https://www.accessdata.fda.gov/drugsatfda _docs/nda/2016/125521Orig1s000ClinPharmR.pdf. Accessed 25 Jul 2018

46. Xu ZH, Lee H, Vu T, Hu C, Yan H, Baker D, et al. Population pharmacokinetics of golimumab in patients with ankylosing spondylitis: impact of body weight and immunogenicity. Int J Clin Pharmacol Ther. 2010;48(9):596-607.

47. Perez Ruixo JJ, Ma P, Chow AT. The utility of modeling and simulation approaches to evaluate immunogenicity effect on the therapeutic protein pharmacokinetics. AAPS J. 2013;15(1):172-82. 